\title{
Meson Masses and Mixing Angles in 2+1 Flavor Polyakov Quark Meson Sigma Model and Symmetry Restoration Effects
}

\author{
Uma Shankar Gupta* and Vivek Kumar Tiwar円 \\ Department of Physics, University of Allahabad, Allahabad 211002, India.
}

(Dated: November 2, 2018)

\begin{abstract}
The meson masses and mixing angles have been calculated for the scalar and pseudoscalar sector in the framework of the generalized $2+1$ flavor Polyakov loop augmented quark meson linear sigma model. We have given the results for two different forms of the effective Polyakov loop potential. The comparison of results with the existing calculations in the bare $2+1$ quark meson linear sigma model, shows that the restoration of chiral symmetry becomes sharper due to the influence of the Polyakov loop potential. We find that inclusion of the Polyakov loop in quark meson linear sigma model together with the presence of axial anomaly, triggers an early and significant melting of the strange condensate. We have examined how the inclusion of the Polyakov loop qualitatively and quantitatively affects the convergence in the masses of the chiral partners in pseudoscalar $\left(\pi, \eta, \eta^{\prime}\right.$, $K)$ and scalar $\left(\sigma, a_{0}, f_{0}, \kappa\right)$ meson nonets as the temperature is varied on the reduced temperature scale. The role of $U_{A}(1)$ anomaly in determining the isoscalar masses and mixing angles for the pseudoscalar $\left(\eta\right.$ and $\eta^{\prime}$ ) and scalar $\left(\sigma\right.$ and $\left.f_{0}\right)$ meson complex, has also been investigated in the Polyakov quark meson linear sigma model. The interplay of chiral symmetry restoration effects and the setting up of $U_{A}(1)$ restoration trend has been discussed and analyzed in the framework of the presented model calculations.
\end{abstract}

PACS numbers: 12.38.Aw, 11.30.Rd, 12.39.Fe, 11.10.Wx

\section{INTRODUCTION}

The present theoretical understanding of strong interaction physics indicates that normal hadronic matter undergoes a phase transition, where the individual hadrons dissolve into their constituents and produce a collective form of matter known as the Quark Gluon Plasma (QGP) under the extreme conditions of high temperature and/or density [1 3$]$. Relativistic heavy ion collision experiments at RHIC (BNL), LHC (CERN) and the future CBM experiments at the FAIR facility (GSI-Darmstadt) aim to create and study such a collective state of matter.

Study of the different aspects of this phase transition, is a tough and challenging task because Quantum Chromodynamics (QCD), the theory of strong interaction, becomes nonperturbative in the low energy limit. In the zero quark mass limit, chiral condensate works as an order parameter for the spontaneous breakdown of the chiral symmetry in the low energy hadronic vacuum of the QCD. For the infinitely heavy quarks, in the pure gauge $S U_{c}(3)$ $\mathrm{QCD}$, the $Z(3)$ (center symmetry of the QCD color gauge group) symmetry, which is the symmetry of hadronic vacuum, gets spontaneously broken in the high temperature/density regime of QGP. Here the expectation value of the Wilson line (Polyakov loop) is related to the free energy of a static color charge, hence it serves as the order parameter of the confinement-deconfinement phase transition [4]. Even though the center symmetry is always broken with the inclusion of dynamical quarks in the system, one can regard the Polyakov loop as an approximate order parameter because it is a good indicator of the confinement-deconfinement transition [5, 6].

We get important information and insights from the lattice QCD calculations (see e.g. 7-14]) regarding various aspects of the transition, like the restoration of chiral symmetry in QCD, order of the confinement-deconfinement phase transition, richness of the QCD phase structure and mapping of the phase diagram. Since lattice calculations are technically involved and various issues are not conclusively settled within the lattice community, one resorts to the calculations within the ambit of phenomenological models developed in terms of effective degrees of freedom. These models serve to complement the lattice simulations and give much needed insight about the regions of phase diagram inaccessible to lattice simulations. A lot of current effective model building activity, is centered around combining the features of spontaneous breakdown of both chiral symmetry as well as the center $Z(3)$ symmetry of QCD in one single model (see for example [15 29]). In these models chiral condensate and Polyakov loop are simultaneously coupled to the quark degrees of freedom.

*Electronic address: guptausg@gmail.com

${ }^{\dagger}$ Electronic address: vivekkrt@gmail.com 
In order to calculate the properties of mesons in hot and dense medium, several investigations have been done in the two and three flavor Nambu-Jona-Lasinio (NJL), Polyakov-Nambu-Jona-Lasinio (PNJL) models (e.g. [30 33]) and also in the $S U(2)$ version of linear sigma model (e.g. 34-36]). Since chiral symmetry restoration is signaled by parity doubling, these studies look for the patterns of emerging convergence in the masses of the chiral partners in pseudoscalar $\left(\pi, \eta, \eta^{\prime}, K\right)$ and scalar mesons $\left(\sigma, a_{0}, f_{0}, \kappa\right)$. It is a common knowledge that the basic QCD Lagrangian has the global $S U_{L+R}(3) \times S U_{L-R}(3) \times U_{A}(1)$ symmetry. Different patterns of spontaneous as well as explicit breaking of $S U_{V}(3) \times S U_{A}(3)$, have been discussed by Lenaghan et al. [37] in the ambit of $S U(3)$ linear sigma model. Schaefer et al. enlarged the linear sigma model with the inclusion of quarks [38] and then they studied in the $2+1$ flavor breaking scenario, the consequences of $S U(3)$ chiral symmetry restoration for scalar and pseudoscalar meson masses and mixing angles, in the presence as well as the absence of $U_{A}(1)$ axial symmetry, as the temperature is increased through the phase transition temperature. The $U_{A}(1)$ axial symmetry does not exist at the quantum level and as shown by 't Hooft [39], it gets explicitly broken to $Z_{A}\left(N_{f}\right)$ by the instanton effects. The $U_{A}(1)$ anomaly does not let the $\eta^{\prime}$ meson remain massless Goldstone boson in the chiral limit by giving it a mass of about $1 \mathrm{GeV}$. This happens due to flavor mixing, a phenomenon that lifts the degeneracy between the $\pi$ and $\eta^{\prime}$ which otherwise would have been degenerate with $\pi$ in $U(3)$ even if the explicit chiral symmetry breaking is present. There is large violation in the Okubo-Zweig-Iizuka rule for both pseudoscalar and scalar mesons and ideal mixing is not achieved because of strong flavor mixing between nonstrange and strange flavor components of the mesons [31]. Hence $U_{A}(1)$ restoration will have important observable effects on scalar and pseudoscalar meson masses as well as the mixing angles.

In a three flavor PNJL model calculation, Costa and collaborators 31] have discussed in detail how the inclusion of Polyakov loop in the NJL model, affects the results of meson mass and mixing angle calculations. However in an earlier paper, they have pointed out that the description of the $\eta^{\prime}$ in the NJL model has some problem [32]. The NJL model does not confine and the meson degrees of freedom are generated in the model by some prescription. The polarization function for the meson gets an imaginary part above the $\bar{q} q$ threshold, hence $\eta^{\prime}$ becomes unbound completely in the model soon after the temperature is raised from zero. Thus $\eta^{\prime}$ in the NJL model is not a well defined quantity [40]. Schaefer et al. 38, 41] have also made an elaborate study of meson masses and mixing angles with and without $U_{A}(1)$ axial anomaly in the $2+1$ flavor quark meson linear sigma model where the mesons are included in the Lagrangian from the very outset and the $U_{A}(1)$ breaking 't Hooft coupling term is constant. The behavior of the scalar and pseudoscalar mixing angles in their calculation is opposite to what has been reported in the calculation by Costa et al. [31]. It is worthwhile and important to investigate the influence of Polyakov loop on meson mass and mixing angle calculations in scalar and pseudoscalar sector, in the framework of generalized $2+1$ flavor quark meson linear sigma model enlarged with the inclusion of the Polyakov loop [42 [44]. Since we are lacking in the experimental information on the behavior of mass and mixing angle observables in the medium, a comparative study of these quantities in different models and circumstances becomes all the more desirable. We will be investigating how the inclusion of Polyakov loop, qualitatively and quantitatively affects the convergence of the masses of chiral partners, when the parity doubling takes place as the temperature is increased through $T_{c}$ and the partial restoration of chiral symmetry is achieved. We will also be studying the effect of Polyakov loop on the interplay of $S U_{A}(3)$ chiral symmetry and $U_{A}(1)$ symmetry restoration.

The arrangement of this paper is as follows. In Sec I we have given the formulation of the model. The description of grand potential in the mean field approach has been presented in Sec. III. We have derived the modification of meson masses due to the $\bar{q} q$ contribution in the presence of Polyakov loop in Sec IV where the formulae for meson

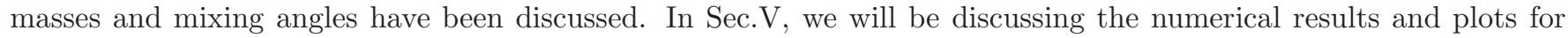
understanding and analyzing the effect of Polyakov loop on chiral symmetry restoration. Discussion and summary is presented in the last SecVI

\section{MODEL FORMULATION}

We will be working in the generalized three flavor quark meson linear sigma model which has been combined with the Polyakov loop potential [42 44]. In this model, quarks coming in three flavor are coupled to the $S U_{V}(3) \times S U_{A}(3)$ symmetric mesonic fields together with spatially constant temporal gauge field represented by Polyakov loop potential. Polyakov loop field $\Phi(\vec{x})$ is defined as the thermal expectation value of color trace of Wilson loop in temporal direction

$$
\Phi=\frac{1}{N_{c}} \operatorname{Tr}_{c} L, \quad \Phi^{*}=\frac{1}{N_{c}} \operatorname{Tr}_{c} L^{\dagger}
$$

where $\mathrm{L}(\mathrm{x})$ is a matrix in the fundamental representation of the $S U_{c}(3)$ color gauge group.

$$
L(\vec{x})=\mathcal{P} \exp \left[i \int_{0}^{\beta} d \tau A_{0}(\vec{x}, \tau)\right]
$$


Here $\mathcal{P}$ is path ordering, $A_{0}$ is the temporal component of Euclidean vector field and $\beta=T^{-1}[4]$.

The model Lagrangian is written in terms of quarks, mesons, couplings and Polyakov loop potential $\mathcal{U}\left(\Phi, \Phi^{*}, T\right)$.

$$
\mathcal{L}_{P Q M S}=\mathcal{L}_{Q M S}-\mathcal{U}\left(\Phi, \Phi^{*}, T\right)
$$

where the Lagrangian in quark meson linear sigma model

$$
\mathcal{L}_{Q M S}=\overline{q_{f}}\left(i \gamma^{\mu} D_{\mu}-g T_{a}\left(\sigma_{a}+i \gamma_{5} \pi_{a}\right)\right) q_{f}+\mathcal{L}_{m}
$$

The coupling of quarks with the uniform temporal background gauge field is effected by the following replacement $D_{\mu}=\partial_{\mu}-i A_{\mu}$ and $A_{\mu}=\delta_{\mu 0} A_{0}$ (Polyakov gauge), where $A_{\mu}=g_{s} A_{\mu}^{a} \lambda^{a} / 2 . g_{s}$ is the $S U_{c}(3)$ gauge coupling. $\lambda_{a}$ are Gell-Mann matrices in the color space, a runs from $1 \cdots 8 . q_{f}=(u, d, s)^{T}$ denotes the quarks coming in three flavors and three colors. $\mathrm{g}$ is the flavor blind Yukawa coupling that couples the three flavor of quarks with nine mesons in the scalar $\left(\sigma_{a}, J^{P}=0^{+}\right)$and pseudoscalar $\left(\pi_{a}, J^{P}=0^{-}\right)$sectors.

The quarks have no intrinsic mass but become massive after spontaneous chiral symmetry breaking because of nonvanishing vacuum expectation value of the chiral condensate. The mesonic part of the Lagrangian has the following form

$$
\begin{aligned}
\mathcal{L}_{m}= & \operatorname{Tr}\left(\partial_{\mu} M^{\dagger} \partial^{\mu} M\right)-m^{2} \operatorname{Tr}\left(M^{\dagger} M\right)-\lambda_{1}\left[\operatorname{Tr}\left(M^{\dagger} M\right)\right]^{2} \\
& -\lambda_{2} \operatorname{Tr}\left(M^{\dagger} M\right)^{2}+c\left[\operatorname{det}(M)+\operatorname{det}\left(M^{\dagger}\right)\right] \\
& +\operatorname{Tr}\left[H\left(M+M^{\dagger}\right)\right] .
\end{aligned}
$$

The chiral field $\mathrm{M}$ is a $3 \times 3$ complex matrix comprising of the nine scalars $\sigma_{a}$ and the nine pseudoscalar $\pi_{a}$ mesons.

$$
M=T_{a} \xi_{a}=T_{a}\left(\sigma_{a}+i \pi_{a}\right)
$$

Here $T_{a}$ represent 9 generators of $U(3)$ with $T_{a}=\frac{\lambda_{a}}{2} . \quad a=0,1 \ldots 8 . \quad \lambda_{a}$ are standard Gell-Mann matrices with $\lambda_{0}=\sqrt{\frac{2}{3}} 1$. The generators follow $U(3)$ algebra $\left[T_{a}, T_{b}\right]=i f_{a b c} T_{c}$ and $\left\{T_{a}, T_{b}\right\}=d_{a b c} T_{c}$ where $f_{a b c}$ and $d_{a b c}$ are standard antisymmetric and symmetric structure constants respectively with $f_{a b 0}=0$ and $d_{a b 0}=\sqrt{\frac{2}{3}} \mathbf{1} \delta_{\mathbf{a b}}$ and matrices are normalized as $\operatorname{Tr}\left(T_{a} T_{b}\right)=\frac{\delta_{a b}}{2}$.

The $S U_{L}(3) \times S U_{R}(3)$ chiral symmetry is explicitly broken by the explicit symmetry breaking term

$$
H=T_{a} h_{a}
$$

Here $\mathrm{H}$ is a $3 \times 3$ matrix with nine external parameters. The $\xi$ field picks up the nonzero vacuum expectation value, $\bar{\xi}$ due to the spontaneous breakdown of the chiral symmetry. Since $\bar{\xi}$ must have the quantum numbers of the vacuum, explicit breakdown of the chiral symmetry is only possible with three nonzero parameters $h_{0}, h_{3}$ and $h_{8}$. We are neglecting isospin symmetry breaking hence we choose $h_{0}, h_{8} \neq 0$. This leads to the $2+1$ flavor symmetry breaking scenario with nonzero condensates $\overline{\sigma_{0}}$ and $\overline{\sigma_{8}}$.

Apart from $h_{0}$ and $h_{8}$, the other parameters in the model are five in number. These are the squared tree-level mass of the meson fields $m^{2}$, quartic coupling constants $\lambda_{1}$ and $\lambda_{2}$, a Yukawa coupling $g$ and a cubic coupling constant $c$ which models the $U_{A}(1)$ axial anomaly of the QCD vacuum.

Since it is broken by the quantum effects, the $U_{A}(1)$ axial which otherwise is a symmetry of the classical Lagrangian, becomes anomalous [45] and gives large mass to $\eta^{\prime}$ meson $\left(m_{\eta^{\prime}}=940 \mathrm{MeV}\right)$. In the absence of $U_{A}(1)$ anomaly, $\eta^{\prime}$ meson would have been the ninth pseudoscalar Goldstone boson, resulting due to the spontaneous break down of the chiral $U_{A}(3)$ symmetry. The entire pseudoscalar nonet corresponding to spontaneously broken $U_{A}(3)$, would consist of the three $\pi$, four $K, \eta$ and $\eta^{\prime}$ mesons, which are the massless pure Goldstone modes when $H=0$ and they become pseudo Goldstone modes after acquiring finite mass due to nonzero $H$ in different symmetry breaking scenarios. The particles coming from octet $\left(a_{0}, f_{0}, \kappa\right)$ and singlet $(\sigma)$ representations of $S U_{V}(3)$ group, constitute scalar nonet $(\sigma$, $\left.a_{0}, f_{0}, \kappa\right)$. In order to study the chiral symmetry restoration at high temperatures, we will be investigating the trend of convergence in the masses of chiral partners occurring in pseudoscalar $\left(\pi, \eta, \eta^{\prime}, K\right)$ and scalar $\left(\sigma, a_{0}, f_{0}, \kappa\right)$ nonets, in the $2+1$ flavor symmetry breaking scenario.

\section{A. Choice of Potentials for the Polyakov Loop}

The effective potential $\mathcal{U}\left(\Phi, \Phi^{*}, T\right)$ is constructed such that it reproduces thermodynamics of pure glue theory on the lattice for temperatures upto about twice the deconfinement phase transition temperature. At much higher 


\begin{tabular}{|l|l||l|l|}
\hline \hline & Scalar Meson Sector & & Pseudoscalar Meson Sector \\
\hline$m_{a_{0}}^{2}$ & $m^{2}+\lambda_{1}\left(x^{2}+y^{2}\right)+\frac{3 \lambda_{2}}{2} x^{2}+\frac{\sqrt{2} c}{2} y$ & $m_{\pi}^{2}$ & $m^{2}+\lambda_{1}\left(x^{2}+y^{2}\right)+\frac{\lambda_{2}}{2} x^{2}-\frac{\sqrt{2} c}{2} y$ \\
$m_{\kappa}^{2}$ & $m^{2}+\lambda_{1}\left(x^{2}+y^{2}\right)+\frac{\lambda_{2}}{2}\left(x^{2}+\sqrt{2} x y+2 y^{2}\right)+\frac{c}{2} x$ & $m_{K}^{2}$ & $m^{2}+\lambda_{1}\left(x^{2}+y^{2}\right)+\frac{\lambda_{2}}{2}\left(x^{2}-\sqrt{2} x y+2 y^{2}\right)-\frac{c}{2} x$ \\
$m_{s, 00}^{2}$ & $m^{2}+\frac{\lambda_{1}}{3}\left(7 x^{2}+4 \sqrt{2} x y+5 y^{2}\right)+\lambda_{2}\left(x^{2}+y^{2}\right)-\frac{\sqrt{2} c}{3}(\sqrt{2} x+y)$ & $m_{p, 00}^{2}$ & $m^{2}+\lambda_{1}\left(x^{2}+y^{2}\right)+\frac{\lambda_{2}}{3}\left(x^{2}+y^{2}\right)+\frac{c}{3}(2 x+\sqrt{2} y)$ \\
$m_{s, 88}^{2}$ & $m^{2}+\frac{\lambda_{1}}{3}\left(5 x^{2}-4 \sqrt{2} x y+7 y^{2}\right)+\lambda_{2}\left(\frac{x^{2}}{2}+2 y^{2}\right)+\frac{\sqrt{2} c}{3}\left(\sqrt{2} x-\frac{y}{2}\right)$ & $m_{p, 88}^{2}$ & $m^{2}+\lambda_{1}\left(x^{2}+y^{2}\right)+\frac{\lambda_{2}}{6}\left(x^{2}+4 y^{2}\right)-\frac{c}{6}(4 x-\sqrt{2} y)$ \\
$m_{s, 08}^{2}$ & $\frac{2 \lambda_{1}}{3}\left(\sqrt{2} x^{2}-x y-\sqrt{2} y^{2}\right)+\sqrt{2} \lambda_{2}\left(\frac{x^{2}}{2}-y^{2}\right)+\frac{c}{3 \sqrt{2}}(x-\sqrt{2} y)$ & $m_{p, 08}^{2}$ & $\frac{\sqrt{2} \lambda_{2}}{6}\left(x^{2}-2 y^{2}\right)-\frac{c}{6}(\sqrt{2} x-2 y)$ \\
$m_{\sigma}^{2}$ & $m_{s, 00}^{2} \cos ^{2} \theta_{s}+m_{s, 88}^{2} \sin ^{2} \theta_{s}+2 m_{s, 08}^{2} \sin \theta_{s} \cos \theta_{s}$ & $m_{\eta^{\prime}}^{2}$ & $m_{p, 00}^{2} \cos ^{2} \theta_{p}+m_{p, 88}^{2} \sin ^{2} \theta_{p}+2 m_{p, 08}^{2} \sin \theta_{p} \cos \theta_{p}$ \\
$m_{f_{0}}^{2}$ & $m_{s, 00}^{2} \sin ^{2} \theta_{s}+m_{s, 88}^{2} \cos ^{2} \theta_{s}-2 m_{s, 08}^{2} \sin \theta_{s} \cos \theta_{s}$ & $m_{\eta}^{2}$ & $m_{p, 00}^{2} \sin ^{2} \theta_{p}+m_{p, 88}^{2} \cos ^{2} \theta_{p}-2 m_{p, 08}^{2} \sin \theta_{p} \cos \theta_{p}$ \\
$m_{\sigma_{N S}}^{2}$ & $\frac{1}{3}\left(2 m_{s, 00}^{2}+m_{s, 88}^{2}+2 \sqrt{2} m_{s, 08}^{2}\right)$ & $m_{\eta_{N S}}^{2}$ & $\frac{1}{3}\left(2 m_{p, 00}^{2}+m_{p, 88}^{2}+2 \sqrt{2} m_{p, 08}^{2}\right)$ \\
$m_{\sigma_{S}}^{2}$ & $\frac{1}{3}\left(m_{s, 00}^{2}+2 m_{s, 88}^{2}-2 \sqrt{2} m_{s, 08}^{2}\right)$ & $m_{\eta_{S}}^{2}$ & $\frac{1}{3}\left(m_{p, 00}^{2}+2 m_{p, 88}^{2}-2 \sqrt{2} m_{p, 08}^{2}\right)$ \\
\hline
\end{tabular}

TABLE I: The squared masses of scalar and pseudoscalar mesons appear in nonstrange-strange basis. In the following table $\mathrm{x}$ denotes $\sigma_{x}$ and y denotes $\sigma_{y}$. The masses of nonstrange $\sigma_{N S}$, strange $\sigma_{S}$, nonstrange $\eta_{N S}$ and strange $\eta_{S}$ mesons are given in the last two rows.

temperatures, the transverse gluons become effective degrees of freedom, hence the construction of effective potential in terms of the Polyakov loop potential is not reliable [18, 30].

At low temperatures, the effective potential $\mathcal{U}\left(\Phi, \Phi^{*}, T\right)$ has only one minimum at $\Phi=0$ in the confined phase. Above the critical temperature for deconfinement transition, $\Phi=0$ becomes metastable local minimum and now, the effective potential has three degenerate global minima at $\Phi \neq 0$ due to the spontaneous breakdown of the $Z(3)$ center symmetry.

In this work, we use the following two choices of the effective potential. The first choice is based on the polynomial expansion in terms of Polyakov loop order parameter $\Phi$ and is given [18] as

$$
\begin{aligned}
\frac{\mathcal{U}_{\mathrm{pol}}\left(\Phi, \Phi^{*}, T\right)}{T^{4}}= & -\frac{b_{2}}{4}\left(|\Phi|^{2}+\left|\Phi^{*}\right|^{2}\right)-\frac{b_{3}}{6}\left(\Phi^{3}+\Phi^{* 3}\right) \\
& +\frac{b_{4}}{16}\left(|\Phi|^{2}+\left|\Phi^{*}\right|^{2}\right)^{2}
\end{aligned}
$$

The second term that is the sum of $\Phi^{3}$ and $\Phi^{* 3}$ terms, causes the three degenerate vacua above the deconfinement phase transition. The potential parameters are adjusted according to the pure gauge lattice data such that the equation of state and Polyakov loop expectation values are reproduced. The temperature dependent coefficient $b_{2}(T)$ governs the confinement-deconfinement phase transition and is given by

$$
b_{2}(T)=a_{0}+a_{1}\left(\frac{T_{0}}{T}\right)+a_{2}\left(\frac{T_{0}}{T}\right)^{2}+a_{3}\left(\frac{T_{0}}{T}\right)^{3} .
$$

The other parameters have the following value

$$
\begin{aligned}
& a_{0}=6.75, \quad a_{1}=-1.95, \quad a_{2}=2.625, \\
& a_{3}=-7.44, \quad b_{3}=0.75, \quad b_{4}=7.5 .
\end{aligned}
$$

The other choice of effective potential as given in Ref. [19], has the logarithmic form. The results produced by this potential are known to be fitted well to lattice results.

$$
\begin{aligned}
\frac{\mathcal{U}_{\log }\left(\Phi, \Phi^{*}, T\right)}{T^{4}}= & -\frac{a(T)}{2} \Phi^{*} \Phi+b(T) \ln \left[1-6 \Phi^{*} \Phi\right. \\
& \left.+4\left(\Phi^{* 3}+\Phi^{3}\right)-3\left(\Phi^{*} \Phi\right)^{2}\right]
\end{aligned}
$$

where the temperature dependent coefficients are as follow

$$
a(T)=a_{0}+a_{1}\left(\frac{T_{0}}{T}\right)+a_{2}\left(\frac{T_{0}}{T}\right)^{2} \quad b(T)=b_{3}\left(\frac{T_{0}}{T}\right)^{3} .
$$

The critical temperature for deconfinement phase transition $T_{0}=270 \mathrm{MeV}$ is fixed for pure gauge sector. The parameters of Eq. (9) are

$$
\begin{array}{ll}
a_{0}=3.51, & a_{1}=-2.47, \\
a_{2}=15.2, & b_{3}=-1.75
\end{array}
$$


Both effective potential fits reproduce equally well the equation of state and the Polyakov loop expectation value.

\begin{tabular}{|c||c|c|c|c|c|c|}
\hline \hline & $\mathrm{C}[\mathrm{MeV}]$ & $m^{2}\left[\mathrm{MeV}^{2}\right]$ & $\lambda_{1}$ & $\lambda_{2}$ & $h_{x}\left[\mathrm{MeV}^{3}\right]$ & $h_{y}\left[\mathrm{MeV}^{3}\right]$ \\
\hline $\mathrm{W} / U_{A}(1)$ & 4807.84 & $(342.52)^{2}$ & 1.40 & 46.48 & $(120.73)^{3}$ & $(336.41)^{3}$ \\
$\mathrm{~W} / \mathrm{o} U_{A}(1)$ & 0 & $-(189.85)^{2}$ & -17.01 & 82.47 & $(120.73)^{3}$ & $(336.41)^{3}$ \\
\hline
\end{tabular}

TABLE II: parameters for $m_{\sigma}=600 \mathrm{MeV}$ with and without $U_{A}(1)$ axial anomaly term.

\section{GRAND POTENTIAL IN THE MEAN-FIELD APPROACH}

The thermodynamics of changing numbers of particles and antiparticles is governed by grand canonical partition function. We are considering a spatially uniform system in thermal equilibrium at finite temperature $\mathrm{T}$ and quark chemical potential $\mu_{f}(f=u, d, s)$. The partition function is written as the path integral over quark/antiquark and meson fields [38]

$$
\begin{aligned}
\mathcal{Z}= & \operatorname{Tr} \exp \left[-\beta\left(\hat{\mathcal{H}}-\sum_{f=u, d, s} \mu_{f} \hat{\mathcal{N}}_{f}\right)\right] \\
= & \int \prod_{a} \mathcal{D} \sigma_{a} \mathcal{D} \pi_{a} \int \mathcal{D} q \mathcal{D} \bar{q} \exp \left[-\int_{0}^{\beta} d \tau \int_{V} d^{3} x\right. \\
& \left.\left(\mathcal{L}_{\mathcal{Q} \mathcal{M} \mathcal{S}}^{\mathcal{E}}+\sum_{f=u, d, s} \mu_{f} \bar{q}_{f} \gamma^{0} q_{f}\right)\right] .
\end{aligned}
$$

where $\mathrm{V}$ is the three dimensional volume of the system, and $\beta=\frac{1}{T}$. For three quark flavors, in general, the three quark chemical potential are different. In this work, we assume that $S U_{V}(2)$ symmetry is preserved and neglect the small difference in masses of $\mathrm{u}$ and $\mathrm{d}$ quarks. Thus the quark chemical potential for $\mathrm{u}$ and $\mathrm{d}$ quarks become equal $\mu_{x}=\mu_{u}=\mu_{d}$. The strange quark chemical potential is $\mu_{y}=\mu_{s}$. Further we consider symmetric quark matter and net baryon number to be zero.

The partition function for the $S U(3)$ version of the linear sigma model with or without quarks can be evaluated by the more advanced many-body resummation techniques such as the self consistent Hartree approximation in the Cornwall, Jackiw and Tomboulis [37, 46] formalism or the so called optimized perturbation theory [47] in its improved version [48 50]. However the predictive power of these methods depends on how they are implemented in different approximation schemes.

In the simple mean field approximation, one does not encounter various problems of the more advanced many-body resummation techniques. In our work, the partition function has been evaluated in the mean-field approximation [35, 38, 41]. We replace meson field by their expectation values $\langle\Phi\rangle=T_{0} \overline{\sigma_{0}}+T_{8} \overline{\sigma_{8}}$ and neglect both thermal as well as quantum fluctuations of meson fields while quarks and antiquarks are retained as quantum field. Now following the standard procedure as given in Refs. [15, 18, 51, 52] one can obtain the expression of grand potential as sum of pure gauge field contribution $\mathcal{U}\left(\Phi, \Phi^{*}, T\right)$, meson contribution and quark/antiquark contribution evaluated in the presence of the Polyakov loop,

$$
\begin{aligned}
\Omega(T, \mu)=-\frac{T \ln Z}{V}= & U\left(\sigma_{0}, \sigma_{8}\right)+\mathcal{U}\left(\Phi, \Phi^{*}, T\right) \\
& +\Omega_{\bar{q} q}(T, \mu)
\end{aligned}
$$

In order to study $2+1$ flavor case, one performs following basis transformation of condensates and external fields from original singlet octet $(0,8)$ basis to nonstrange strange basis $(\mathrm{x}, \mathrm{y})$.

$$
\begin{aligned}
\sigma_{x} & =\sqrt{\frac{2}{3}} \bar{\sigma}_{0}+\frac{1}{\sqrt{3}} \bar{\sigma}_{8}, \\
\sigma_{y} & =\frac{1}{\sqrt{3}} \bar{\sigma}_{0}-\sqrt{\frac{2}{3}} \bar{\sigma}_{8} .
\end{aligned}
$$


Similar expressions exist for writing the external fields $\left(h_{x}, h_{y}\right)$ in terms of $\left(h_{0}, h_{8}\right)$. Thus the nonstrange and strange quark/antiquark decouple and the quark masses become

$$
m_{x}=g \frac{\sigma_{x}}{2}, \quad m_{y}=g \frac{\sigma_{y}}{\sqrt{2}}
$$

Quarks become massive in symmetry broken phase because of non zero vacuum expectation values of the condensates.

The mesonic potential in the nonstrange-strange basis reads,

$$
\begin{aligned}
U\left(\sigma_{x}, \sigma_{y}\right)= & \frac{m^{2}}{2}\left(\sigma_{x}^{2}+\sigma_{y}^{2}\right)-h_{x} \sigma_{x}-h_{y} \sigma_{y}-\frac{c}{2 \sqrt{2}} \sigma_{x}^{2} \sigma_{y} \\
& +\frac{\lambda_{1}}{2} \sigma_{x}^{2} \sigma_{y}^{2}+\frac{1}{8}\left(2 \lambda_{1}+\lambda_{2}\right) \sigma_{x}^{4} \\
& +\frac{1}{8}\left(2 \lambda_{1}+2 \lambda_{2}\right) \sigma_{y}^{4},
\end{aligned}
$$

The chiral part of the Polyakov loop augented quark meson linear sigma (PQMS) model has the six input parameters and therefore require six known quantities as input. In general $m_{\pi}, m_{K}$, the pion and kaon decay constant $f_{\pi}, f_{K}$, mass square of $\eta, \eta^{\prime}$ and $m_{\sigma}$ are used to fix these parameters. The parameters are fitted such that in vacuum the model produces observed pion mass $138 \mathrm{MeV}$. In the present work we are using the set of parameters for sigma mass $m_{\sigma}=600 \mathrm{MeV}$. The parameters used in this work, taken from [38], are shown in Table

Finally the quark/antiquark Polyakov loop contribution reads,

$$
\Omega_{\bar{q} q}(T, \mu)=-2 T \sum_{f=u, d, s} \int \frac{d^{3} p}{(2 \pi)^{3}}\left[\ln g_{f}^{+}+\ln g_{f}^{-}\right]
$$

We define $g_{f}^{+}$and $g_{f}^{-}$after taking trace over color space

$$
\begin{aligned}
& g_{f}^{+}=\left[1+3 \Phi e^{-E_{f}^{+} / T}+3 \Phi^{*} e^{-2 E_{f}^{+} / T}+e^{-3 E_{f}^{+} / T}\right] \\
& g_{f}^{-}=\left[1+3 \Phi^{*} e^{-E_{f}^{-} / T}+3 \Phi e^{-2 E_{f}^{-} / T}+e^{-3 E_{f}^{-} / T}\right]
\end{aligned}
$$

Here we use the notation $\mathrm{E}_{f}^{ \pm}=E_{f} \mp \mu$ and $E_{f}$ is the flavor dependent single particle energy of quark/antiquark.

$$
E_{f}=\sqrt{p^{2}+m_{f}^{2}}
$$

$m_{f}$ is flavor dependent quark mass and is function of condensates $\sigma_{0}$ and $\sigma_{8}$.

One can very easily notice from equations (17) and (18) that the role of quarks and antiquarks as well as that of the Polyakov loop and its conjugate can be interchanged by the transformation $\mu \rightarrow-\mu$. Confinement is the very interesting feature of the QCD and the PQMS model describes this behavior qualitatively. The Polyakov loop is order parameter for confinement-deconfinement phase transition. In the confined phase $\Phi=0$. It can also be noticed from the grand potential that one and two quark state contributions are vanishing. Only the three quark states contribute. In this way the PQMS model qualitatively mimics confinement of quark/antiquark within three quark color singlet states [22].

One can get the quark condensates $\sigma_{x}, \sigma_{y}$ and the Polyakov loop expectation values $\Phi, \Phi^{*}$ by searching the global minima of the grand potential for a given value of temperature $\mathrm{T}$ and chemical potential $\mu$.

$$
\frac{\partial \Omega}{\partial \sigma_{x}}=\frac{\partial \Omega}{\partial \sigma_{y}}=\frac{\partial \Omega}{\partial \Phi}=\left.\frac{\partial \Omega}{\partial \Phi^{*}}\right|_{\sigma_{x}=\bar{\sigma}_{x}, \sigma_{y}=\bar{\sigma}_{y}, \Phi=\bar{\Phi}, \Phi^{*}=\bar{\Phi}^{*}}=0 .
$$

In this work we are always considering the $\mu=0$ case. 


\section{MESON MASSES AND MIXING ANGLES}

The curvature of grand potential Eq.(11) at the global minimum determines scalar and pseudoscalar meson masses.

$$
m_{\alpha, a b}^{2}=\left.\frac{\partial^{2} \Omega(T, \mu)}{\partial \xi_{\alpha, a} \partial \xi_{\alpha, b}}\right|_{\min }
$$

where subscript $\alpha=\mathrm{s}$, p; s stands for scalar and $\mathrm{p}$ stands for pseudoscalar meson and $\mathrm{a}, b=0 \cdots 8$. We note that the Polyakov loop decouples from the mesonic sector at $\mathrm{T}=0$ and the meson masses do not receive contribution from quark/antiquark in vacuum and hence meson masses are governed by mesonic potential only. The mesonic contribution to the meson masses is summarized in Table II The diagonalization of $(0-8)$ component of mass matrix gives masses of $\sigma$ and $f_{0}$ mesons in scalar sector and masses of $\eta^{\prime}$ and $\eta$ in pseudoscalar sector. The scalar mixing angle $\theta_{s}$ and pseudoscalar mixing angle $\theta_{p}$ are given by,

$$
\tan 2 \theta_{\alpha}=\left(\frac{2 m_{\alpha, 08}^{2}}{m_{\alpha, 00}^{2}-m_{\alpha, 88}^{2}}\right)
$$

Here $\alpha$ stands for scalar and pseudoscalar field. The detail expressions for masses and mixing angles are given in Ref. [37, 38]. The meson masses are further modified in medium at finite temperature by the quark contributions in the grand potential. In order to calculate the second derivative Eq.(21) for evaluating the quark contribution in the presence of the Polyakov loop potential, the complete dependence of all scalar and pseudoscalar meson fields Eq. (6) has to be taken into account. We have to diagonalize the resulting quark mass matrix. The expression for the meson mass modification due to quark contribution at finite temperature in QMS model, has been evaluated by Schaefer et al. [38] and is given as

$$
\begin{aligned}
\delta m_{\alpha, a b}^{2}= & \left.\frac{\partial^{2} \Omega_{\bar{q} q}(T, \mu)}{\partial \xi_{\alpha, a} \partial \xi_{\alpha, b}}\right|_{\text {min }}=\nu_{c} \sum_{f=x, y} \int \frac{d^{3} p}{(2 \pi)^{3}} \frac{1}{2 E_{f}} \\
& {\left[\left(a_{f}^{+}+a_{f}^{-}\right)\left(m_{f, a b}^{2}-\frac{m_{f, a}^{2} m_{f, b}^{2}}{2 E_{f}^{2}}\right)\right.} \\
& \left.-\left(b_{f}^{+}+b_{f}^{-}\right)\left(\frac{m_{f, a}^{2} m_{f, b}^{2}}{2 E_{f} T}\right)\right]
\end{aligned}
$$

$m_{f, a}^{2} \equiv \partial m_{f}^{2} / \partial \xi_{\alpha, a}$ is the first derivative and $m_{f, a b}^{2} \equiv \partial m_{f, a}^{2} / \partial \xi_{\alpha, b}$ is the second derivative of squared quark mass with respect to meson fields $\xi_{\alpha, b}$. The number of internal quark degrees of freedom, $\nu_{c}=2 N_{c}=6$. Here $a_{f}^{ \pm}$are quark/antiquark occupation numbers; given as

$$
a_{f}^{ \pm}=\frac{1}{1+e^{E^{ \pm} / T}}
$$

\begin{tabular}{|cc|cccc|}
\hline \hline & $m_{x, a}^{2} m_{x, b}^{2} / g^{4}$ & $m_{x, a b}^{2} / g^{2}$ & $m_{y, a}^{2} m_{y, b}^{2} / g^{4}$ & $m_{y, a b}^{2} / g^{2}$ \\
\hline$\sigma_{0}$ & $\sigma_{0}$ & $\frac{1}{3} \sigma_{x}^{2}$ & $\frac{2}{3}$ & $\frac{1}{3} \sigma_{y}^{2}$ & $\frac{1}{3}$ \\
$\sigma_{1}$ & $\sigma_{1}$ & $\frac{1}{2} \sigma_{x}^{2}$ & 1 & 0 & 0 \\
$\sigma_{4}$ & $\sigma_{4}$ & 0 & $\sigma_{x} \frac{\sigma_{x}+\sqrt{2} \sigma_{y}}{\sigma_{x}^{2}-2 \sigma_{y}^{2}}$ & 0 & $\sigma_{y} \frac{\sqrt{2} \sigma_{x}+2 \sigma_{y}}{2 \sigma_{y}^{2}-\sigma_{x}^{2}}$ \\
$\sigma_{8}$ & $\sigma_{8}$ & $\frac{1}{6} \sigma_{x}^{2}$ & $\frac{1}{3}$ & $\frac{2}{3} \sigma_{y}^{2}$ & $\frac{2}{3}$ \\
$\sigma_{0}$ & $\sigma_{8}$ & $\frac{\sqrt{2}}{6} \sigma_{x}^{2}$ & $\frac{\sqrt{2}}{3}$ & $-\frac{\sqrt{2}}{3} \sigma_{y}^{2}$ & $-\frac{\sqrt{2}}{3}$ \\
$\pi_{0}$ & $\pi_{0}$ & 0 & $\frac{2}{3}$ & 0 & $\frac{1}{3}$ \\
$\pi_{1}$ & $\pi_{1}$ & 0 & 1 & 0 & 0 \\
$\pi_{4}$ & $\pi_{4}$ & 0 & $\sigma_{x} \frac{\sigma_{x}-\sqrt{2} \sigma_{y}}{\sigma_{x}^{2}-2 \sigma_{y}^{2}}$ & 0 & $\sigma_{y} \frac{\sqrt{2} \sigma_{x}-2 \sigma_{y}}{\sigma_{x}^{2}-2 \sigma_{y}^{2}}$ \\
$\pi_{8}$ & $\pi_{8}$ & 0 & $\frac{1}{3}$ & 0 & $\frac{2}{3}$ \\
$\pi_{0}$ & $\pi_{8}$ & 0 & $\frac{\sqrt{2}}{3}$ & 0 & $-\frac{\sqrt{2}}{3}$ \\
\hline
\end{tabular}

TABLE III: First and second derivative of squared quark mass in nonstrange-strange basis with respect to meson fields are evaluated at minimum. Sum over two light flavors, denoted by symbol x, are in third and fourth columns. The last two columns have only strange quark mass flavor denoted by the symbol y. 
and the notations $b_{f}^{ \pm}=a_{f}^{ \pm}-\left(a_{f}^{ \pm}\right)^{2}$ stand for particle $(+)$ and antiparticle $(-)$ in quark meson linear sigma model without inclusion of the Polyakov loop.

The expression of mass modification due to quark contribution at finite temperature, will change in the presence of the Polyakov loop. We are obtaining the following formula for the mass modification that results on account of quark contribution in the PQMS model

$$
\begin{aligned}
\delta m_{\alpha, a b}^{2}= & \left.\frac{\partial^{2} \Omega_{\bar{q} q}(T, \mu)}{\partial \xi_{\alpha, a} \partial \xi_{\alpha, b}}\right|_{\text {min }}=3 \sum_{f=x, y} \int \frac{d^{3} p}{(2 \pi)^{3}} \frac{1}{E_{f}} \\
& {\left[\left(A_{f}^{+}+A_{f}^{-}\right)\left(m_{f, a b}^{2}-\frac{m_{f, a}^{2} m_{f, b}^{2}}{2 E_{f}^{2}}\right)\right.} \\
& \left.+\left(B_{f}^{+}+B_{f}^{-}\right)\left(\frac{m_{f, a}^{2} m_{f, b}^{2}}{2 E_{f} T}\right)\right]
\end{aligned}
$$

The notations $A_{f}^{ \pm}$and $B_{f}^{ \pm}$have the following definitions

$$
\begin{aligned}
& A_{f}^{+}=\frac{\Phi e^{-E_{f}^{+} / T}+2 \Phi^{*} e^{-2 E_{f}^{+} / T}+e^{-3 E_{f}^{+} / T}}{g_{f}^{+}} \\
& A_{f}^{-}=\frac{\Phi^{*} e^{-E_{f}^{-} / T}+2 \Phi e^{-2 E_{f}^{-} / T}+e^{-3 E_{f}^{-} / T}}{g_{f}^{-}}
\end{aligned}
$$

and $B_{f}^{ \pm}=3\left(A_{f}^{ \pm}\right)^{2}-C_{f}^{ \pm}$, where we again define

$$
\begin{aligned}
& C_{f}^{+}=\frac{\Phi e^{-E_{f}^{+} / T}+4 \Phi^{*} e^{-2 E_{f}^{+} / T}+3 e^{-3 E_{f}^{+} / T}}{g_{f}^{+}} \\
& C_{f}^{-}=\frac{\Phi^{*} e^{-E_{f}^{-} / T}+4 \Phi e^{-2 E_{f}^{-} / T}+3 e^{-3 E_{f}^{-} / T}}{g_{f}^{-}}
\end{aligned}
$$

The squared quark mass derivatives evaluated at minimum which were originally derived in Ref. [38], are collected in Table TIII The inclusion of Polyakov loop in QMS model does not make any change in these equations.

\section{EFFECT OF THE POLYAKOV LOOP ON THE RESTORATION OF CHIRAL SYMMETRY}

We are presenting the result of our calculation for estimating the effect of the Polyakov loop potential on the restoration of chiral symmetry when it is included in the $2+1$ flavor quark meson linear sigma model at finite temperature and zero chemical potential with and without axial $U_{A}(1)$ breaking. We have considered the two different ansatzs for the Polyakov loop potential namely the polynomial potential and logarithmic potential and compared the results with the existing calculations in the quark meson linear sigma model [38]. The interplay of the effect of $U_{A}(1)$ axial restoration and chiral symmetry restoration in the presence of the Polyakov loop potential has been shown through the temperature variation of strange, nonstrange chiral condensates, meson masses and mixing angles. The $U_{A}(1)$ axial breaking term has been kept constant throughout the investigation. The value of Yukawa coupling g has been fixed from the nonstrange constituent quark mass $m_{q}=300 \mathrm{MeV}$ and is equal to 6.5. This predicts the strange quark mass $m_{s} \simeq 433 \mathrm{MeV}$.

\begin{tabular}{|c|c|c|c|}
\hline & QMS & PQMS:pol & PQMS:log \\
\hline$T_{c}^{\chi}(\mathrm{MeV})$ & 146 & 204 & 206 \\
$T_{s}^{\chi}(\mathrm{MeV})$ & 248 & 262 & 274 \\
$T_{c}^{\Phi}(\mathrm{MeV})$ & - & 204 & 206 \\
\hline
\end{tabular}

TABLE IV: The characteristic temperature (pseudocritical temperature) for the chiral transition in the nonstrange sector $T_{c}^{\chi}$, strange sector $T_{s}^{\chi}$ and confinement-deconfinement transition $T_{c}^{\Phi}$, in the QMS, PQMS:log and PQMS:pol models. 


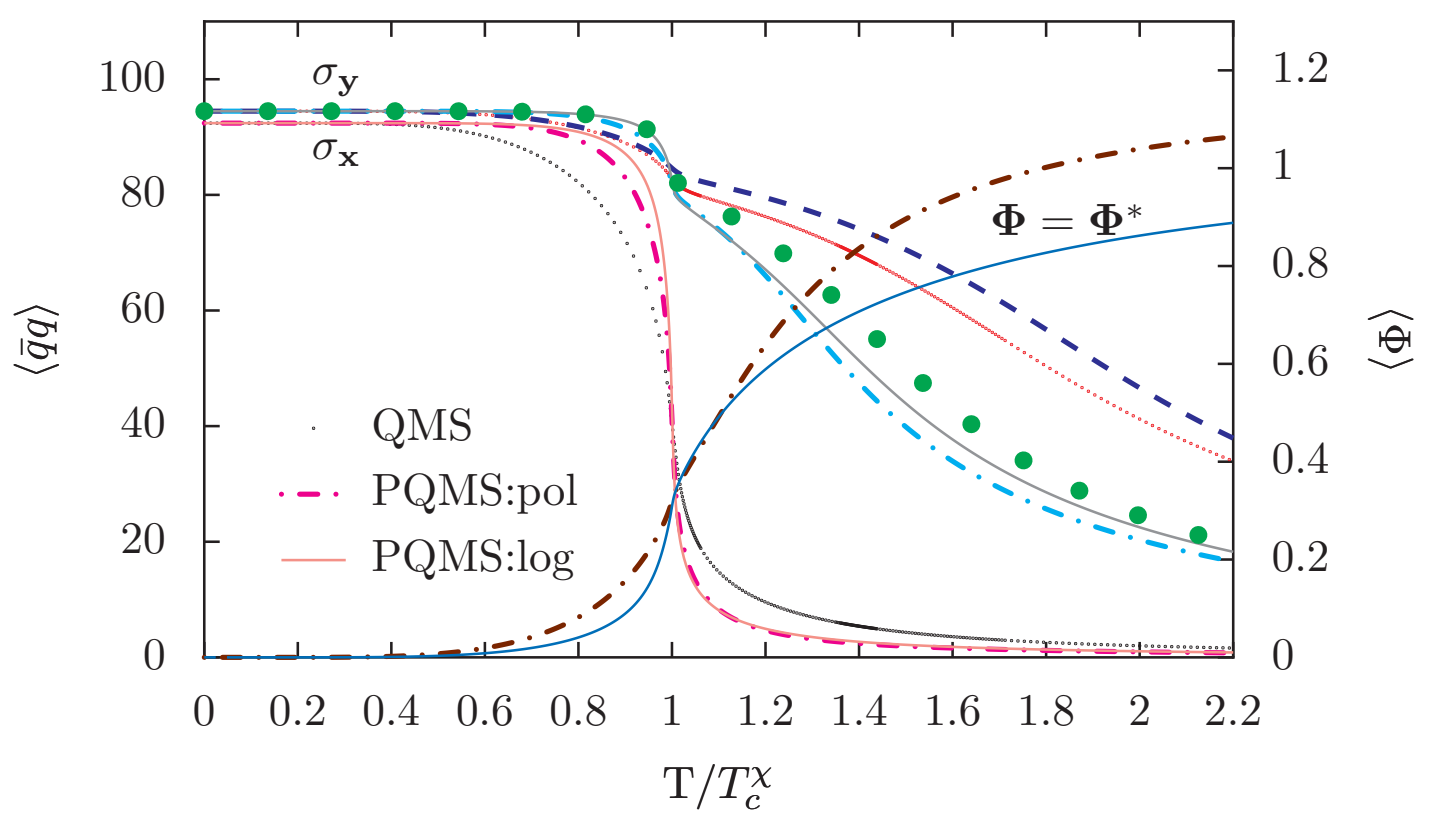

FIG. 1: The variation of nonstrange $\sigma_{x}$, strange $\sigma_{y}$ condensates with respect to the relative temperature scale $\left(T / T_{c}^{\chi}\right)$ at zero chemical potential $(\mu=0)$ in the QMS model and PQMS models with polynomial and logarithmic potentials for the Polyakov loop is shown. The lines with continuous dots represent the variation in the QMS model, while the dashed-dotted lines show the variation in PQMS:pol model and the solid lines are the variations in the PQMS:log model. The line with the big solid dots shows the $\sigma_{y}$ variation in the PQMS:log model, while the dark dashed line shows the pure QMS model results when anomaly is absent, i.e. $c=0$. The expectation value of the Polyakov loop $\langle\Phi\rangle$, in PQMS:pol and PQMS:log model is shown in the right plots.

\section{A. Condensates and the Polyakov Loop}

The solutions of the coupled gap equations, Eq.(20) determine the nature of chiral and deconfinement phase transition through the temperature and chemical potential dependence of nonstrange and strange condensates $\left(\sigma_{x}\right.$ and $\left.\sigma_{y}\right)$ and the expectation value of the Polyakov loop $\left(\langle\Phi\rangle\right.$ and $\left.\left\langle\Phi^{*}\right\rangle\right)$. The temperature variation of $\sigma_{x}, \sigma_{y}$ and $\langle\Phi\rangle$ in mean-field approximation, at zero chemical potential in the PQMS models with the polynomial Polyakov loop potential Eq.(8) (PQMS:pol) and the logarithmic Polyakov loop potential Eq.(9) (PQMS:log) is shown in Fig1] We have also plotted the strange and nonstrange condensate in QMS model to compare and investigate the effect of the Polyakov loop potential inclusion on chiral symmetry restoration trend reflected through masses and mixing angles of mesonic excitations. The characteristic temperatures (pseudocritical temperature) for the confinement deconfinement transition $T_{c}^{\Phi}$, the chiral transition in the nonstrange sector $T_{c}^{\chi}$ and strange sector $T_{s}^{\chi}$ are defined through the inflection point of $\langle\Phi\rangle, \sigma_{x}$, and $\sigma_{y}$. We note that the $\langle\Phi\rangle=\left\langle\Phi^{*}\right\rangle$ at zero chemical potential. The numerical value of the pseudocritical temperature for various transitions in the QMS model and the PQMS model with the polynomial and the logarithmic potentials for the Polyakov loop has been given in Table V It is evident from the table that the chiral transition gets shifted to the higher temperatures as a result of the inclusion of the Polyakov loop potential in the QMS model.

We have chosen to compare the results of our calculation in the PQMS model with the corresponding results in the QMS model on a relative temperature scale $T / T_{c}^{\chi}$. Such a choice is justified on account of the Ginzburg-Landau effective theory, where absolute comparison of the characteristic temperatures between two models of the same universality class can not be made [31]. The condensates start with fixed values $\sigma_{x}=92.4 \mathrm{MeV}$ and $\sigma_{y}=94.5$ $\mathrm{MeV}$ at $T=0$ as shown in Figl. It is known from the lattice simulations that transition from hadronic matter to quark gluon plasma is a analytic and rapid crossover $([7,[10])$. The Polyakov loop potential inclusion in the QMS models makes the crossover in $S U_{L}(2) \times S U_{R}(2)$ sector quite sharp as the nonstrange condensate $\sigma_{x}$ changes rapidly in the transition region. The $U_{A}(1)$ anomaly does not cause any difference in the behavior of nonstrange condensate and $\sigma_{x}$ remain unchanged in the presence as well as in the absence of $U_{A}(1)$ anomaly term. The variation of the strange condensate is lot more smooth on account of the large constituent mass of the strange quark $m_{s}=433 \mathrm{MeV}$. The Polyakov loop potential inclusion has a strong effect on the strange condensate variation also and generates a significant melting of $\sigma_{y}$ in our calculation. The interesting physical consequences of the earlier and significant melting 

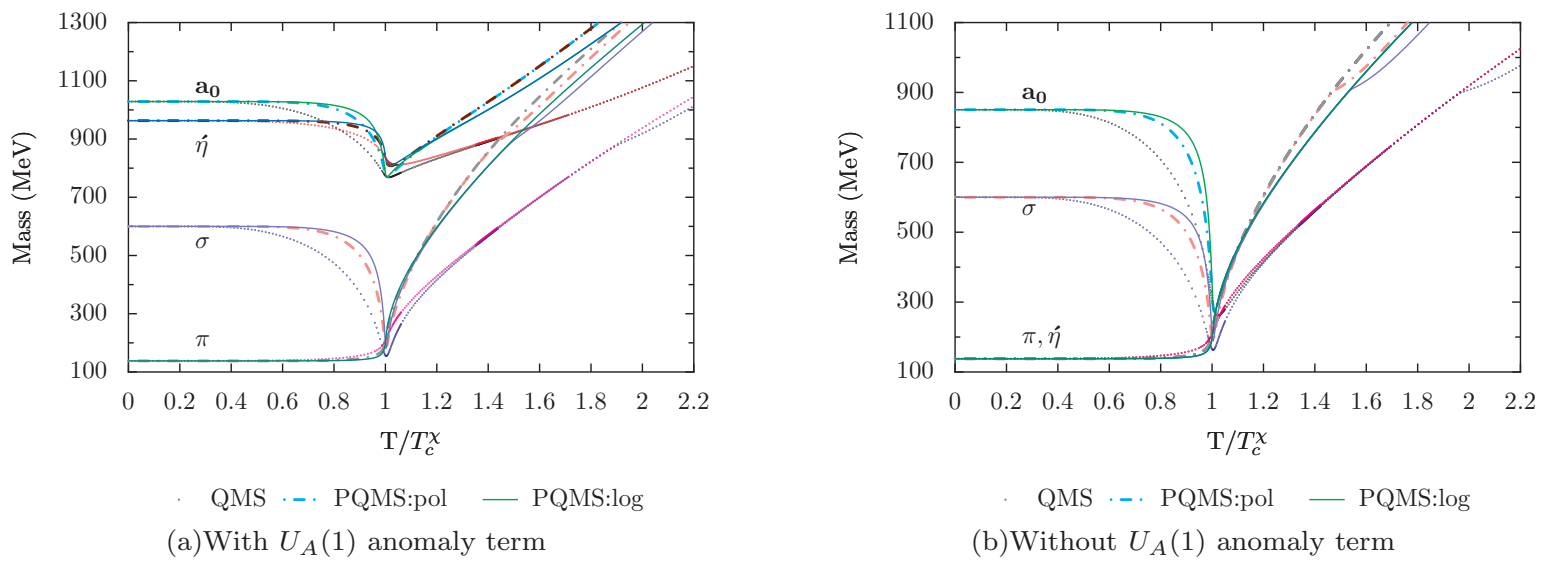

(b)Without $U_{A}(1)$ anomaly term

FIG. 2: The mass variations of the chiral partners as functions of reduced temperature $\left(T / T_{c}^{\chi}\right)$ at zero chemical potential $(\mu=0)$, in the presence of axial $U_{A}(1)$ breaking term, are plotted for $(\sigma, \pi)$ and $\left(a_{0}, \eta^{\prime}\right)$ in Fig $2(\mathrm{a})$ and the corresponding mass variations, in the absence of the $U_{A}(1)$ axial breaking term, are plotted in Fig 2(b) The dotted line plots are the mass variations in the pure QMS model, dashed- dotted line plots represent the PQMS:pol model results, and the solid line plots are the mass variations in the PQMS:log model.

of the strange condensate will be an early emergence of mass degeneration trend in the masses of the chiral partners $(K, \kappa)$ and $\left(\eta, f_{0}\right)$ and an early setting up of a $U_{A}(1)$ restoration trend on reduced temperature scale. In the presence of the $U_{A}(1)$ anomaly, $\sigma_{y}$ temperature variation shows a little more decrease in the respective cases.

Curves starting from the right end of the plot represent the variation of the Polyakov loop expectation value $\langle\Phi\rangle$ on the relative temperature scale at zero chemical potential. Though the thermodynamics of quark gluon plasma reproduced with the Polyakov loop polynomial potential is found to be in agreement with that of lattice simulations, upto twice of the critical temperature $([15,18,42])$ at higher temperatures $\langle\Phi\rangle$ increases above unity and this is unphysical. In the improved ansatz, logarithmic potential replaces the higher order terms of $\Phi$ and $\Phi^{*}$ in the polynomial potential by the logarithm of Jacobi determinant which results from integrating out six nondiagonal Lie algebra directions while keeping the two diagonal ones [19, 52] and thus the logarithmic divergence avoids an expectation value higher than one. This means that the logarithmic potential describes the dynamics of gluons more correctly and effectively. Keeping this in mind, we will be mainly focusing on the discussion of the results in our calculation with the inclusion of the logarithmic Polyakov loop potential, though the curves of the calculation with the polynomial Polyakov loop potential will also be shown. The real physical effect of the Polyakov loop potential inclusion in the QMS model on mesonic excitations, will become apparent when the results of our calculation in the PQMS models are compared with the corresponding results in the QMS model.

\section{B. Meson Mass Variations}

We are calculating the masses of the scalar and pseudoscalar mesons at finite temperature in the presence of the Polyakov loop potential in the QMS model. We have collected the vacuum value of all the scalar and pseudoscalar meson masses in Table II The mass modifications calculated at finite temperature (Eq 25) will be added to the vacuum masses of Table [I] The mass variations of the chiral partners as functions of reduced temperature, in the presence of axial $U_{A}(1)$ breaking term, are plotted for $(\sigma, \pi)$ and $\left(a_{0}, \eta^{\prime}\right)$ in Fig $2(\mathrm{a})$ and for $\left(\eta, f_{0}\right)$ and $(K, \kappa)$ in Fig 3(a), while the corresponding mass variations, in the absence of the $U_{A}(1)$ axial breaking term, are plotted in Fig 2(b) and Fig 3(b) Further, since the focus of our investigation is the influence of the Polyakov loop on the effective restoration of symmetries, we will be comparing the mesonic observables below and above $T_{c}^{\chi}$.

In Fig 2(a), the chiral partners $(\sigma, \pi)$ and $\left(a_{0}, \eta^{\prime}\right)$ become mass degenerate in the close vicinity of reduced temperature $T / T_{c}^{\chi}=1$. The masses of these particles are dominated by the contribution from the nonstrange quarks and a rapid crossover in the nonstrange sector (Fig \) appears as sharper and faster mass degeneration in our calculation in the PQMS model. Thus, the Polyakov loop inclusion in the QMS model makes a sharper mass degeneration as well as faster occurrence of chiral $S U_{L}(2) \times S U_{R}(2)$ symmetry restoration transition in the nonstrange sector.

In Fig $3(\mathrm{a})$, the presence of the Polyakov loop potential in the QMS model generates, the similar trend of sharper and faster mass degeneration in the masses of the chiral partners $\left(\eta, f_{0}\right)$ and $(K, \kappa)$. Though the mass degeneration of chiral partners $(K, \kappa)$ with $\eta$ does not occur at $T / T_{c}^{\chi}=1$, it sets up early in the PQMS models at $T / T_{c}^{\chi}=1.3$, 

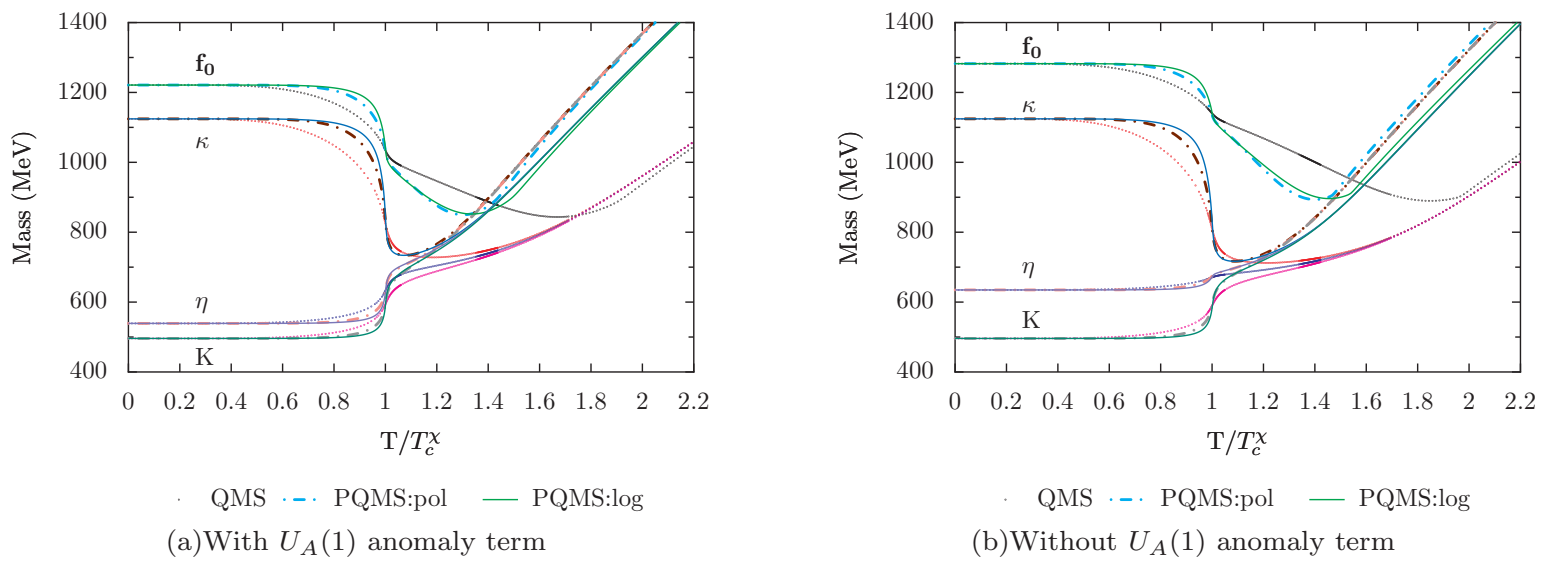

(b) Without $U_{A}(1)$ anomaly term

FIG. 3: The mass variations of the chiral partners as functions of reduced temperature $\left(T / T_{c}^{\chi}\right)$ at zero chemical potential $(\mu=0)$, in the presence of axial $U_{A}(1)$ breaking term, are shown for $\left(\eta, f_{0}\right)$ and $(K, \kappa)$ in Fig $3(\mathrm{a})$ and the corresponding mass variations, in the absence of the $U_{A}(1)$ axial breaking term, are shown in Fig $3(\mathrm{~b})$ The dotted line plots are the mass variations in the pure QMS model, dashed-dotted line plots represent the PQMS:pol model results, and the solid line plots are the mass variations in the PQMS:log model.

while it occurs at $T / T_{c}^{\chi}=1.5$ in the QMS model. In the PQMS models, the intersection point of the $f_{0}$ and $\eta$ masses, occurs early when $T / T_{c}^{\chi}=1.4$, while in the QMS model it is found at $T / T_{c}^{\chi}=1.7$. This trend of mass degeneration reflects the effect of the Polyakov loop potential on chiral symmetry restoration in the strange sector and it results due to sharper and stronger melting of the strange condensate (Fig 1) in the influence of the Polyakov loop potential in the PQMS models.

The $U_{A}(1)$ breaking generates the mass gap between the two sets of the chiral partners, $(\sigma, \pi)$ and $\left(a_{0}, \eta^{\prime}\right)$, i.e. $m_{\pi}=m_{\sigma}<m_{a_{0}}=m_{\eta^{\prime}}$ for $T / T_{C}^{\chi}>1$. This mass gap results due to the opposite sign of the anomaly term $\left(\sqrt{2} c \sigma_{y}\right)$ in the scalar and pseudoscalar meson masses. Hence, it will be reduced due to the melting of the strange order parameter $\sigma_{y}$ for the higher values of the reduced temperature $T / T_{c}^{\chi}>1$. Since the melting of the strange condensate is stronger and sharper (Fig (1) in the PQMS model, the convergence in the masses of the two sets of chiral partners gets enhanced in these calculations. Thus the inclusion of the Polyakov loop potential in the QMS model also effects an early set up of $U_{A}(1)$ restoration trend on the reduced temperature scale.

Now we discuss the variations in the masses of chiral partners when the explicit $U_{A}(1)$ symmetry breaking term has been taken as zero $(c=0)$. We notice in Fig 2(b) and Fig 3(b), again, the same sharper and faster trend of mass degeneration that we identify as the effect generated by the inclusion of the Polyakov loop potential. The $\eta^{\prime}$ meson degenerates with the pion in vacuum and stays the same for all temperatures in Fig 2(b) due to the absence of the anomaly term. Further the mass gap between the chiral partners $(\sigma, \pi)$ and $\left(a_{0}, \eta^{\prime}\right)$ becomes zero and all four of the mesons become degenerate at $T / T_{c}^{\chi}=1.0$. The $T / T_{c}^{\chi}$ numerical value, where the $K, \kappa$, and $\eta$ masses degenerate in different models, is not influenced by the $U_{A}(1)$ anomaly as expected since the nonstrange condensate does not have any anomaly dependence. Further,in Fig $3(\mathrm{~b})$, the intersection point of the $f_{0}$ and $\eta$ masses in the PQMS models, is obtained when $T / T_{c}^{\chi}$ is around 1.6 while in the QMS model, this intersection point is found around $T / T_{c}^{\chi}=2.0$. We are also obtaining the mild anomaly dependence of the intersection point of the $f_{0}$ and $\eta$ in all the models. Here, we note that the mass of $f_{0}$ in vacuum increases by about $60 \mathrm{MeV}$ in the absence of anomaly.

The temperature variations of meson masses, in general, result due to the interplay of the bosonic thermal contributions (decreasing the meson masses) and fermionic quark contributions (increasing the meson masses). Quark contributions which are negligible at small temperatures, dominate the mesonic contributions for high temperatures, and this generates a rising trend in meson masses, which ultimately leads to the mass degeneration of the chiral partners [38]. In the PQMS models, the one quark and two quark fermionic contributions are suppressed due to the presence of the Polyakov loop potential. Since the chiral phase transition is driven by the fermionic contributions, chiral restoration gets delayed due to the delay in the deconfinement transition and because of this we get higher value of the pseudocritical temperature $T_{c}^{\chi}$ in the Polyakov loop augmented quark meson linear sigma model. The higher value of the $T_{c}^{\chi}$ makes the ratio $T / T_{c}^{\chi}$ small. Hence, in comparison to the QMS model, the mass degeneration trend among the chiral partners, in general, sets up early in the PQMS models on the reduced temperature scale.

The variation of meson masses with the polynomial Polyakov loop potential are similar though a little less sharp than the mass calculations with the logarithmic potential. The difference appears mainly because of difference in the Polyakov loop expectation value $\langle\Phi\rangle$ with these two potentials. The calculations with the polynomial Polyakov loop 


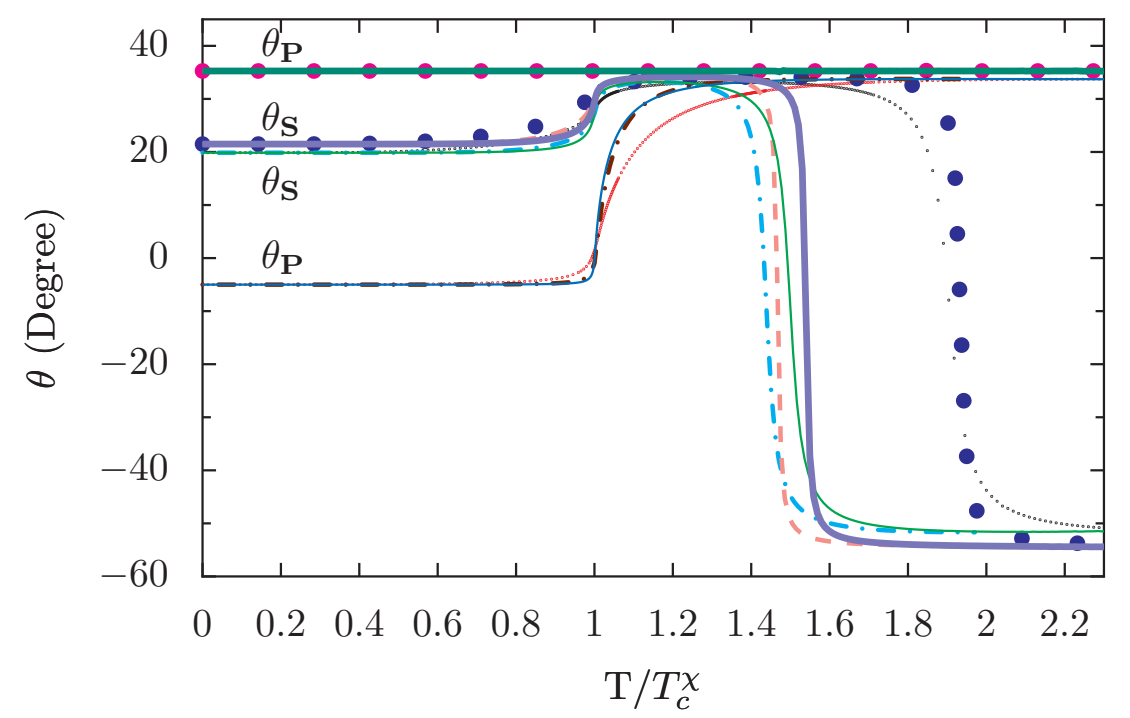

$$
\begin{aligned}
& \text { QMS w/ } U_{A}(1) \quad \cdots \text { PQMS:pol w/ } U_{A}(1) \quad \longrightarrow \text { PQMS:log w/ } U_{A}(1) \\
& \text { - } \text { QMS w/o } U_{A}(1) \quad \text { - - PQMS:pol w/o } U_{A}(1) \quad \text { PQMS:log w/o } U_{A}(1)
\end{aligned}
$$

FIG. 4: The scalar $\theta_{S}$ and pseudoscalar $\theta_{P}$ mixing angle variations with respect to the reduced temperature $\left(T / T_{c}^{\chi}\right)$ at zero chemical potential $(\mu=0)$ are plotted. We have given the plots for the QMS, PQMS:log, and PQMS:pol models considering the cases in the presence as well as absence of the axial $U_{A}(1)$ explicit symmetry breaking term. The dotted lines show the result with anomaly in the QMS model while the solid big dot lines show the result without anomaly. In the PQMS:log model, the thick solid lines represent the variations without anomaly while thin solid lines show the result with anomaly. The dashed-dotted lines are the variations with anomaly in the PQMS:pol model, while the dashed lines are the corresponding results in the absence of anomaly.

potential make sense only for $T<2 T_{c}^{\chi}$.

The mass variation of scalar $\sigma$ and $f_{0}$ show kink around $T / T_{c}^{\chi}=1.8$ in the QMS model while it is seen around $T / T_{c}^{\chi}=1.4$ in the PQMS models. The kink generation results because the meson masses seem to interchange their identities for higher values of the reduced temperature [38]. In order to have a proper perspective of the kink behavior in the curves, one has to study and analyze the scalar and pseudoscalar meson mixing angles.

\section{Meson Mixing Angle Variations}

The analysis of axial $U_{A}(1)$ restoration pattern identification will become complete, only after studying the variation of scalar $\theta_{S}$ and pseudoscalar $\theta_{P}$ mixing angles on the relative temperature scale in Fig 4 considering the cases in the presence as well as the absence of the axial $U_{A}(1)$ explicit symmetry breaking term. The anomaly term has a strong effect in the pseudoscalar sector in the broken phase for $T / T_{c}^{\chi}<1$ while no effect of anomaly is found in the scalar sector. The nonstrange and strange quark mixing is strong, at $\mathrm{T}=0$ one gets $\theta_{P}=-5^{\circ}$, which remains almost constant in the chiral broken phase. In the vicinity of $T / T_{C}^{\chi}=1$, the $\theta_{P}$ variations start approaching the ideal mixing angle $\theta_{P} \rightarrow \arctan \frac{1}{\sqrt{2}} \sim 35^{\circ}$, the corresponding $\Phi_{P}=90^{\circ}$. Here, $\Phi_{P}$ is the pseudoscalar mixing angle in the strange nonstrange basis (see Ref. 38] for details). The smooth approach towards the ideal mixing in the QMS model, becomes sharper and faster in the PQMS model calculations due to the influence of the Polyakov loop potential. Further, the ideal mixing is achieved earlier on the reduced temperature scale in the PQMS models. In the absence of axial $U_{A}(1)$ anomaly, the pseudoscalar mixing angle remains ideal $\theta_{P}=35^{\circ}$ everywhere on the reduced temperature scale.

The $\eta$ and $\eta^{\prime}$ mesons become a purely strange $\eta_{S}$ and nonstrange $\eta_{N S}$ quark system as a consequence of the ideal pseudoscalar mixing, which gets fully achieved at higher values of the reduced temperature. In order to show this, we have plotted in Fig $5(\mathrm{a})$ the mass variations for the physical $\eta, \eta^{\prime}$ and the nonstrange-strange $\eta_{N S}, \eta_{S}$ complex. Mass formulae $m_{\eta_{N S}}$ and $m_{\eta_{S}}$ are given in Table II Again, the smooth mass convergence trend, of the pure QMS model in $m_{\eta^{\prime}} \rightarrow m_{\eta_{N S}}$ and $m_{\eta} \rightarrow m_{\eta_{S}}$ approach, becomes sharper and faster around $T / T_{c}^{\chi}=1$ in the influence of the Polyakov loop potential in the QMS model. The exact $m_{\eta^{\prime}} \rightarrow m_{\eta_{N S}}$ and $m_{\eta} \rightarrow m_{\eta_{S}}$ mass convergence in the PQMS models, occurs closer to the value $T / T_{c}^{\chi}=1$. 


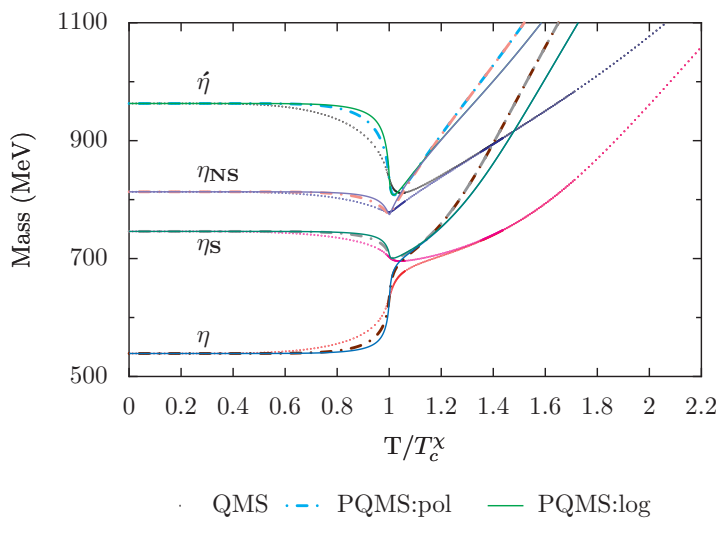

(a)With $U_{A}(1)$ anomaly term

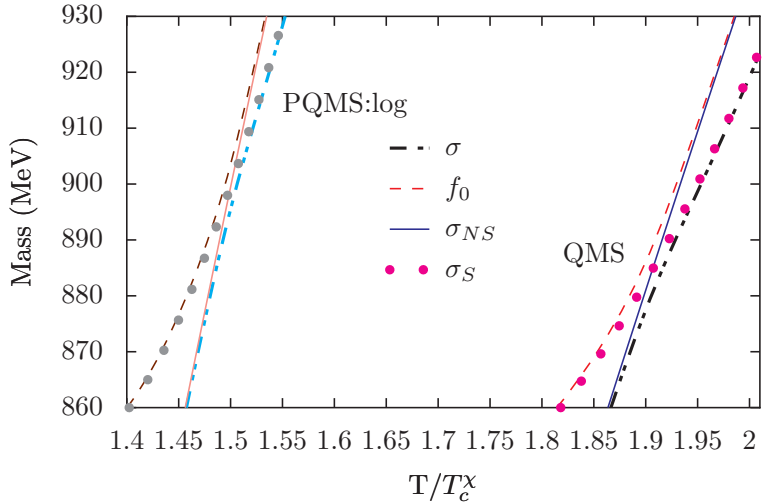

(b)With $U_{A}(1)$ anomaly term.

FIG. 5: Figure5(a) shows the mass variations for the physical $\eta, \eta^{\prime}$ and the nonstrange-strange $\eta_{N S}$, $\eta_{S}$ complex, on the reduced temperature scale $\left(T / T_{c}^{\chi}\right)$ at zero chemical potential $(\mu=0)$. The masses of the physical $\sigma$ and $f_{0}$ anticross and the nonstrange-strange $\sigma_{N S}-\sigma_{S}$ system masses cross in Figur $5(\mathrm{~b})$

In Fig 凹for $m_{\sigma}=600 \mathrm{MeV}$ at $\mathrm{T}=0$ scalar mixing angle $\theta_{S} \sim 19.9^{\circ}$ in the presence of anomaly, while $\theta_{S} \sim 21.5^{\circ}$ in the absence of anomaly. The $\theta_{S}$ around $T / T_{c}^{\chi}=1$ grows to its ideal value but for higher temperatures on the reduced temperature scale, in the chiral symmetric phase, the scalar mixing angle drops down to $\theta_{S} \sim-51^{\circ}$ and $\theta_{S} \sim-54^{\circ}$ in the respective cases considered with and without anomaly. In the presence of the $U_{A}(1)$ symmetry breaking term, this drop happens in the QMS model around $T / T_{c}^{\chi} \sim 1.9$ and due to the effect of the Polyakov loop potential the similar drop occurs earlier for $T / T_{c}^{\chi} \sim 1.5$ in the PQMS model. In the close vicinity of these reduced temperatures, the masses of the physical $\sigma$ and $f_{0}$ anticross and the nonstrange - strange $\left(\sigma_{N S}-\sigma_{S}\right)$ system masses cross as shown in Fig 5(b) It means that after anticrossing the physical $\sigma$ becomes identical with the pure strange quark system $\sigma_{S}$, while the physical $f_{0}$ becomes degenerate with the pure nonstrange quark system $\sigma_{N S}$. A similar drop for the calculations without anomaly happens at a little higher value on the reduced temperature scale in respective models.

\section{SUMMARY AND DISCUSSION}

We have calculated the meson masses and mixing angles for the scalar and pseudoscalar sector in the framework of the generalized 2+1 flavor PQMS model. We have used two different forms of the effective Polyakov loop potential for the calculation, namely, the polynomial potential and logarithmic potential. In order to investigate the influence of Polyakov loop potential on chiral symmetry restoration, these calculations have been compared with the corresponding results in the QMS model.

The temperature dependence of nonstrange, strange condensates and the Polyakov loop field $\Phi$ at zero chemical potential has been calculated from the gap equation in the QMS and PQMS models. Comparison of pseudocritical temperatures calculated from the inflection points of these order parameters indicates, that the chiral transition gets shifted to the higher temperatures as a result of the inclusion of the Polyakov loop in the QMS model. We further observe that the variation of the nonstrange condensate in the $T / T_{c}^{\chi}=0.8$ to 1.2 range becomes quite sharp due to the effect of the Polyakov loop potential in our calculation in PQMS models. We infer from the curves in the PQMS models that the inclusion of the Polyakov loop potential in the QMS model together with the presence of axial anomaly, triggers an early and significant melting of the strange condensate. The interesting physical consequences of the earlier melting of the strange condensate are an early emergence of mass degeneration trend in the masses of the chiral partners $(K, \kappa)$ and $\left(\eta, f_{0}\right)$ and an early setting up of a $U_{A}(1)$ restoration trend.

The mass degeneration of chiral partners $(\sigma, \pi)$ and $\left(a_{0}, \eta^{\prime}\right)$ in the close vicinity of $T / T_{c}^{\chi}=1.0$ becomes sharper and faster in our calculations in the PQMS model. This sharpening of the mass variations in the small neighborhood of $T / T_{c}^{\chi}=1$ results due to the stronger and sharper melting of the nonstrange condensate triggered by the presence of the Polyakov loop potential in the QMS model. Thus, we can corroborate also from the behavior of the chiral partners that the net effect of the Polyakov loop inclusion in the QMS model, is to make a sharper occurrence of the chiral $S U(2)_{L} \times S U(2)_{R}$ symmetry restoration transition in the nonstrange sector. Further, the mass degeneration of chiral partners $(K, \kappa)$ with $\eta$ does not occur when the value of the reduced temperature is equal to one, it sets up early in the PQMS models at $T / T_{c}^{\chi}=1.3$, while it occurs at $T / T_{c}^{\chi}=1.5$ in the QMS model. In the PQMS models, the intersection point of the $f_{0}$ and $\eta$ masses, occurs early when the reduced temperature $T / T_{c}^{\chi}=1.3$, while in the 
pure QMS model this intersection point is found at $T / T_{c}^{\chi}=1.7$. This trend of mass degeneration emerges, again as a result of the sharper and stronger melting of the strange condensate in the influence of the Polyakov loop potential in the PQMS models.

The $U_{A}(1)$ breaking anomaly effect that leads to the mass gap between the two sets of the chiral partners, $(\sigma, \pi)$ and $\left(a_{0}, \eta^{\prime}\right)$ i.e. $m_{\pi}=m_{\sigma}<m_{a_{0}}=m_{\eta^{\prime}}$ for $T / T_{C}^{\chi}>1$, is proportional to the strange condensate $\sigma_{y}$. Since the melting of the strange condensate is stronger and sharper in the PQMS models, the convergence in the masses of the two sets of chiral partners will be enhanced in these calculations. Thus, the inclusion of the Polyakov loop potential in the PQMS models also effects an early set up of the $U_{A}(1)$ restoration trend on the reduced temperature scale.

The smooth approach of the pseudoscalar mixing angle $\theta_{P}$ towards the ideal mixing in the QMS model, becomes sharper and faster in the PQMS models due to the influence of the Polyakov loop potential. Further, in comparison to QMS model results, the ideal mixing on the reduced temperature scale is achieved earlier in the PQMS models. The $\theta_{S}$ around $T / T_{c}^{\chi}=1$ grows to its ideal value but for higher temperatures on the reduced temperature scale, in the chirally symmetric phase, the scalar mixing angle drops down to $\theta_{S} \sim-51^{\circ}$. In the presence of $U_{A}(1)$ symmetry breaking term, this drop happens in the QMS model for $T / T_{c}^{\chi} \sim 1.85$ and in the PQMS:log model, the similar drop occurs for $T / T_{c}^{\chi} \sim 1.5$. In the close vicinity of these reduced temperatures, the masses of the physical $\sigma$ and $f_{0}$ anticross and the nonstrange-strange $\sigma_{N S}-\sigma_{S}$ system masses cross.

\section{Acknowledgments}

We are very thankful to Ajit Mohan Srivastava, Tamal K. Mukherjee and Neelima Agarwal for valuable discussions. We acknowledge the support of the Department of Atomic Energy- Board of Research in Nuclear Sciences (DAE-BRNS), India, under the research grant No. 2008/37/13/BRNS. We also acknowledge the computational support of the computing facility which has been developed by the Nuclear Particle Physics group of the Physics Department, Allahabad University under the Center of Advanced Studies(CAS) funding of UGC India.

[1] L.D.McLerran, B.Svetitsky, Phys. Rev. D24, 450 (1981); B.Svetitsky, Phys. Rep. 132, 1 (1986).

[2] D. H. Rischke, Prog. Part. Nucl. Phys. 52, 197 (2004).

[3] B.Muller, Rep. Prog. Phys. 58, 611 (1995).

[4] A. M. Polyakov, Phys. Lett. B 72, 477 (1978).

[5] R. D. Pisarski, Phys. Rev. D 62 111501(R) (2000).

[6] B. Layek, A. P. Mishra, A. M. Srivastava and V. K. Tiwari, Phys. Rev. D 73103514 (2006).

[7] F. Karsch, Lect. Notes Phys. 583, 209 (2002).

[8] Z. Fodor, S. D. Katz, and K. K. Szabo, Phys. Lett. B 568, 73 (2003).

[9] C. R. Allton, M. Doring, S. Ejiri, S. J. Hands, O. Kaczmarek, F. Karsch, E Laermann and K. Redlich, Phys. Rev. D 71, $054508(2005)$.

[10] Y. Aoki, Z. Fodor, S. D. Katz and K. K. Szabo, Phys. Lett. B 643, 46 (2006).

[11] F. Karsch, J. Phys. G 31, S633 (2005).

[12] F. Karsch, e-Print: arXiv:0701.210 [hep-ph].

[13] M. Cheng et al., Phys. Rev. D 74, 054507 (2006).

[14] M. Cheng et al., Phys. Rev. D 77, 014511 (2008).

[15] B. J. Schaefer, J. M. Pawlowski and J. Wambach, Phys. Rev. D 76074023 (2007)

[16] S. Digal, E. Laermann and H. Satz, Eur. Phys. J. C 18583 (2001).

[17] T. Kähärä and K. Tuominen Phys. Rev. D 78034015 (2008)

[18] C. Ratti, M. A. Thaler and W. Weise, Phys. Rev. D 73, 014019 (2006).

[19] S. Rößner, C. Ratti, and W. Weise, Phys. Rev. D 75, 034007 (2007).

[20] S. Rößner, T. Hell, C. Ratti, and W. Weise, Nucl. Phys. A 814118 (2008).

[21] S. K. Ghosh, T. K. Mukherjee, M. G. Mustafa and R. Ray, Phys. Rev. D 73, 114007 (2006).

[22] C. Sasaki, B. Friman and K. Redlich, Phys. Rev D 75, 074013 (2007).

[23] T. Hell, S. Rößner, M. Cristoforetti and W. Weise, Phys. Rev D 79, 014022 (2009).

[24] H. Abuki, R. Anglani, R. Gatto, G. Nardulli and M. Ruggieri, Phys. Rev D 78, 034034 (2008).

[25] M. Ciminale, R. Gatto, N. D. Ippolito, G. Nardulli and M. Ruggieri, Phys. Rev D 77, 054023 (2008).

[26] W.-J. Fu, Z. Zhang and Y.-X. Liu, Phys. Rev D 77, 014006 (2008).

[27] K. Fukushima, Phys. Rev D 77, 114028 (2008).

[28] K. Fukushima, Phys. Rev D 78, 114019 (2008).

[29] K. Fukushima, Phys. Rev D 79, 074015 (2009).

[30] H. Hansen, W. M. Alberico, A. Beraudo, A Molinari, M. Nardi and C. Ratti Phys. Rev. D 75, 065004 (2007).

[31] P. Costa, M. C. Ruivo, C. A. de Sousa, H. Hansen and W. M. Alberico Phys. Rev. D 79, 116003 (2009). 
[32] P. Costa, M. C. Ruivo, C. A. de Sousa and Yu. L. Kalinovsky Phys. Rev. D 71, 116002 (2005).

[33] P. Costa, M. C. Ruivo, C. A. de Sousa and Yu. L. Kalinovsky Phys. Rev. D 70, 116013 (2004).

[34] B. J. Schaefer and J. Wambach, Phys. Rev. D 75085015 (2007)

[35] O. Scavenius, A. Mocsy, I. N. Mishustin, D. H. Rischke, Phys. Rev. C 64, 045202 (2001).

[36] J. Schaffner-Bielich, Phys. Rev. Lett. 84, 3261 (2000).

[37] J. T. Lenaghan, D. H. Rischke and J. Schaffner-Bielich, Phys. Rev D 62, 085008 (2000).

[38] B. J. Schaefer and M. Wagner, Phys. Rev. D 79014018 (2009).

[39] G. 't Hooft, Phys. Rev. Lett. 37, 8 (1976); Phys. Rev D 14, 3432 (1976).

[40] K. Fukushima, K. Ohnishi, K. Ohta, Phys. Rev C 63, 045203 (2001).

[41] B. J. Schaefer and M. Wagner, arXiv:0812.2855 [hep-ph].

[42] B. J. Schaefer, M. Wagner and J. Wambach, e-Print: arXiv:0910.5628 [hep-ph]

[43] B. J. Schaefer, M. Wagner and J. Wambach, e-Print: arXiv:0909.0289 [hep-ph]

[44] H. Mao, J. Jin and M. Huang, J. Phys. G 37, 035001 (2010).

[45] S. Weinberg Phys. Rev. D 11, 3583 (1975).

[46] J. M. Cornwall, R. Jackiw and E. Tomboulis, Phys. Rev D $102428(1974)$.

[47] S. Chiku and T. Hatsuda, Phys. Rev. D 58076001 (1998).

[48] T. Herpay, A. Patkós, Zs. Szép and P. Szépfalusy, Phys. Rev. D 71125017 (2005).

[49] T. Herpay and Zs. Szép, Phys. Rev. D 74025008 (2006).

[50] P. Kovács and Zs. Szép, Phys. Rev. D 75025015 (2007).

[51] Finite Temperature Field Theory Principles and Applications, J. I. Kapusta and C. Gale, Cambridge University Press, Cambridge, England (2006).

[52] K. Fukushima, Phys. Lett. B 591277 (2004). 